\title{
A CENTRAL LIMIT THEOREM FOR REVERSIBLE PROCESSES WITH NONLINEAR GROWTH OF VARIANCE
}

\author{
OU ZHAO, ${ }^{*}$ University of South Carolina \\ MICHAEL WOODROOFE, ${ }^{* *}$ University of Michigan \\ DALIBOR VOLNÝ, ${ }^{* * *}$ Université de Rouen
}

\begin{abstract}
Kipnis and Varadhan (1986) showed that, for an additive functional, $S_{n}$ say, of a reversible Markov chain, the condition $\mathrm{E}\left[S_{n}^{2}\right] / n \rightarrow \kappa \in(0, \infty)$ implies the convergence of the conditional distribution of $\left.S_{n} / \sqrt{\mathrm{E}\left[S_{n}^{2}\right.}\right]$, given the starting point, to the standard normal distribution. We revisit this question under the weaker condition, $\mathrm{E}\left[S_{n}^{2}\right]=n \ell(n)$, where $\ell$ is a slowly varying function. It is shown by example that the conditional distributions of $\left.S_{n} / \sqrt{\mathrm{E}\left[S_{n}^{2}\right.}\right]$ need not converge to the standard normal distribution in this case; and sufficient conditions for convergence to a (possibly nonstandard) normal distribution are developed.
\end{abstract}

Keywords: Conditional distribution; Markov chain; self-adjoint operator; slowly varying function.

2010 Mathematics Subject Classification: Primary 60F05

Secondary 60J05

\section{Introduction}

Consider a reversible Markov chain $\ldots, W_{-1}, W_{0}, W_{1}, \ldots$, defined on a probability space $(\Omega, \mathcal{A}, \mathrm{P})$, with a Polish state space $\mathcal{W}$, transition function $Q$, and marginal distribution $\pi$. Thus, $\pi\{B\}=\mathrm{P}\left[W_{n} \in B\right], Q(w ; B)=\mathrm{P}\left[W_{n+1} \in B \mid W_{n}=w\right]$, and (the reversibility condition)

$$
\int_{A} Q(w ; B) \pi\{\mathrm{d} w\}=\int_{B} Q(w ; A) \pi\{\mathrm{d} w\}
$$

for Borel sets $A, B \subseteq \mathcal{W}, w \in \mathcal{W}$, and $n \in \mathbb{Z}$. Using (and abusing) notation in a standard manner, we write

$$
Q f(w)=\int_{W} f(z) Q(w ; \mathrm{d} z) \quad \pi \text {-almost everywhere }
$$

for $f \in L^{1}(\pi)$ and $Q^{k}=Q \circ \cdots \circ Q$ for the iterates of $Q$. In addition, let $L_{0}^{p}(\pi)=\{f \in$ $\left.L^{p}(\pi): \int_{W} f \mathrm{~d} \pi=0\right\}$,

$$
V_{n}=I+Q+\cdots+Q^{n-1}, \quad \text { and } \quad \bar{V}_{n}=\frac{1}{n}\left(V_{1}+\cdots+V_{n}\right),
$$

Received 3 November 2009; revision received 26 May 2010.

* Postal address: Department of Statistics, University of South Carolina, 1523 Greene Street, Columbia, SC 29208, USA. Email address: ouzhao@stat.sc.edu

** Postal address: Department of Statistics and Mathematics, University of Michigan, 275 West Hall, 1085 South University, Ann Arbor, MI 48109, USA. Email address: michaelw@umich.edu

*** Postal address: Laboratoire de Mathématiques Raphaël Salem, UMR CNRS 6085, Université de Rouen, France. Email address: dalibor.volny@univ-rouen.fr 
and let $\|\cdot\|$ denote the norm in an $L^{2}$ space, either $L^{2}(\pi)$ or $L^{2}(\mathrm{P})$. Finally, ' $\stackrel{\mathrm{D}}{\rightarrow}$ ' denotes convergence in distribution and $\stackrel{\mathrm{P}}{\rightarrow}$ ' denotes convergence in probability of conditional distributions; that is, if the $Z_{n}: \Omega \rightarrow \mathbb{R}$ are random variables and $G$ is a distribution function, then $Z_{n} \mid W_{0} \stackrel{\mathrm{P}}{\rightarrow} G$ means that the conditional distribution of $Z_{n}$ given $W_{0}$ converges in probability to $G$.

The reversibility condition (1) is equivalent to requiring that $\left(W_{0}, W_{1}\right)$ and $\left(W_{1}, W_{0}\right)$ have the same distribution, since the left-hand side of (1) is $\mathrm{P}\left[W_{0} \in A, W_{1} \in B\right]$ and the righthand side is $\mathrm{P}\left[W_{0} \in B, W_{1} \in A\right]$. An important consequence (also equivalent) is that the restriction of $Q$ to $L^{2}(\pi)$ is a self-adjoint operator. For $\langle f, g\rangle=\int_{W} f g \mathrm{~d} \pi$, where $\langle\cdot, \cdot\rangle$ denotes the inner product in $L^{2}(\pi),\langle f, Q g\rangle=\mathrm{E}\left[f\left(W_{0}\right) g\left(W_{1}\right)\right]=\mathrm{E}\left[f\left(W_{1}\right) g\left(W_{0}\right)\right]=\langle Q f, g\rangle$ for all $f, g \in L^{2}(\pi)$.

Given $g \in L_{0}^{2}(\pi)$, let $X_{k}=g\left(W_{k}\right), S_{n}=X_{1}+\cdots+X_{n}$, and $\sigma_{n}^{2}=\mathrm{E}\left[S_{n}^{2}\right]$. Kipnis and Varadhan [7] showed that if

$$
\lim _{n \rightarrow \infty} \frac{\sigma_{n}^{2}}{n}=\kappa \in[0, \infty)
$$

then the conditional distribution of $S_{n} / \sqrt{n}$ given $W_{0}$ converges in probability to the normal distribution with mean 0 and variance $\kappa$. We show in Proposition 1 that $\kappa>0$ except for trivial special cases; then $\sigma_{n}^{-1} S_{n} \mid W_{0} \stackrel{\text { P }}{\rightarrow} \operatorname{Normal}[0,1]$. Kipnis and Varadhan showed that $S_{n}$ could be written in the form $S_{n}=M_{n}+R_{n}$, where $0=M_{0}, M_{1}, M_{2}, \ldots$ is a square integrable martingale with (strictly) stationary increments $D_{k}=M_{k}-M_{k-1}$ and $\left\|R_{n}\right\|=o(\sqrt{n})$. The result has applications to Monte Carlo Markov chains (see, for instance, [12]), since many algorithms lead to reversible chains, and, to interacting particle systems [6], [7].

Here we consider the case in which (2) is weakened to

$$
\sigma_{n}^{2}=n \ell(n)
$$

where $\ell$ is a slowly varying function, as defined in Chapter 1 of [2]. An example will show that the main result from [7] does not extend completely. Some features do extend, however. For the remainder of the paper, reversibility is assumed along with $g \in L_{0}^{2}(\pi)$, and $\ell$ is defined by (3).

Further developments under condition (2) may be found in [3], and [10] is a recent article on asymptotic normality of sums of stationary processes with nonlinear growth of variance.

\section{Generalities}

In the first proposition below, we show that the case $\lim _{n \rightarrow \infty} \ell(n)=\infty$ only needs to be considered. The relation

$$
\sigma_{n}^{2}=\left[2\left\langle g, \bar{V}_{n} g\right\rangle-\|g\|^{2}\right] n
$$

is used in its proof.

Proposition 1. If $\liminf _{n \rightarrow \infty} \ell(n)<\infty$ then (2) holds, and if $\liminf _{n \rightarrow \infty} \ell(n)=0$ then $S_{n}=\frac{1}{2}\left[1+(-1)^{n-1}\right] X_{1}$ with probability 1 .

Proof. Since $Q$ is self-adjoint, we may write $Q=\int_{\Lambda} \lambda \mathrm{d} M(\lambda)$, where $\Lambda \subseteq[-1,1]$ is the spectrum of $Q$ and $M$ is a countably additive, projection-valued set function defined on the Borel sets of $\Lambda$. Then $Q^{k}=\int_{\Lambda} \lambda^{k} \mathrm{~d} M(\lambda)$ for all $k \geq 1$. See [5, Chapter 2]. Let $\mu_{g}(B)=\langle g, M(B) g\rangle$. Then $\mu_{g}$ is a measure, and

$$
\left\langle g, \bar{V}_{n} g\right\rangle=\int_{\Lambda}\left(1-\frac{\lambda}{n} \frac{1-\lambda^{n}}{1-\lambda}\right) \frac{\mu_{g}(\mathrm{~d} \lambda)}{1-\lambda} .
$$


Observe that the integrand on the right-hand side of (5) is nonnegative. So, if $\lim _{\inf } n \rightarrow \infty \ell(n)$ $<\infty$ then the limit inferior of the left-hand side of (5) is finite and, therefore,

$$
\int_{\Lambda} \frac{\mu_{g}(\mathrm{~d} \lambda)}{1-\lambda}<\infty
$$

by Fatou's lemma. It is clear the integrands on the right-hand side of (5) are dominated by an integrable function; hence, the integral converges to that on the left-hand side of (6), and (2) holds with

$$
\kappa=2 \int_{\Lambda} \frac{\mu_{g}(\mathrm{~d} \lambda)}{1-\lambda}-\|g\|^{2}=\int_{\Lambda} \frac{1+\lambda}{1-\lambda} \mu_{g}(\mathrm{~d} \lambda) .
$$

If $\liminf _{n \rightarrow \infty} \ell(n)=0$ then the last integral is 0 and, therefore, $\mu_{g}$ is a point mass at $\{-1\}$. It follows that $Q g=-g, \mathrm{E}\left[\left(X_{0}+X_{1}\right)^{2}\right]=0, X_{n}=(-1) X_{n-1}$ with probability 1 , and $S_{n}=\frac{1}{2}\left[1+(-1)^{n-1}\right] X_{1}$ with probability 1 .

As a consequence, there is no loss of generality in supposing that $\ell(n) \rightarrow \infty$, which we do where convenient. For if $\lim _{n \rightarrow \infty} \ell(n)<\infty$ then the Kipnis-Varadhan result is applicable.

The proof of the next proposition uses (4) and

$$
\bar{V}_{n}=\sum_{k=0}^{n-1}\left(1-\frac{k}{n}\right) Q^{k}
$$

Proposition 2. If $\ell$ varies slowly in (3) then $\left\|V_{n} g\right\|=o\left(\sigma_{n}\right)$.

Proof. Using the reversibility and (7),

$$
\begin{aligned}
\left\|V_{n} g\right\|_{2}^{2} & =\sum_{j=0}^{n-1} \sum_{k=0}^{n-1}\left\langle g, Q^{k+j} g\right\rangle \\
& =\sum_{i=0}^{n-1}(i+1)\left\langle g, Q^{i} g\right\rangle+\sum_{i=n}^{2 n-2}(2 n-1-i)\left\langle g, Q^{i} g\right\rangle \\
& =\sum_{i=0}^{2 n-2}(2 n-1-i)\left\langle g, Q^{i} g\right\rangle-2 \sum_{i=0}^{n-1}(n-1-i)\left\langle g, Q^{i} g\right\rangle \\
& =\frac{1}{2}\left[\sigma_{2 n-1}^{2}+(2 n-1)\|g\|^{2}\right]-\left[\sigma_{n-1}^{2}+(n-1)\|g\|^{2}\right] \\
& =\frac{1}{2} \sigma_{2 n-1}^{2}-\sigma_{n-1}^{2}+\frac{1}{2}\|g\|^{2} .
\end{aligned}
$$

The proposition then follows directly from (3) and the slow variation of $\ell$.

Corollary 1. If $\ell$ varies slowly then there is a sequence of square integrable martingales $0=M_{n, 1}, M_{n, 2}, \ldots$ with stationary increments $D_{n, k}=M_{n, k}-M_{n, k-1}, k \geq 1$, for which $\max _{k \leq n}\left\|S_{k}-M_{n, k}\right\|=o\left(\sigma_{n}\right)$.

Proof. The result follows from Proposition 2 and Theorem 1 of [13]. It is relevant that

$$
D_{n, k}=\bar{V}_{n} g\left(W_{k}\right)-Q \bar{V}_{n} g\left(W_{k-1}\right)
$$

and $M_{n, k}=D_{n, 1}+\cdots+D_{n, k}$ in the proof of the latter result. 
Corollary 2. If $\ell$ varies slowly and there is a $\lambda \geq 0$ for which

$$
\frac{1}{\sigma_{n}^{2}} \sum_{k=1}^{n} \mathrm{E}\left[D_{n, k}^{2} \mid W_{k-1}\right] \rightarrow^{\mathrm{P}} \lambda
$$

and

$$
\frac{1}{\sigma_{n}^{2}} \sum_{k=1}^{n} \mathrm{E}\left[D_{n, k}^{2} \mathbf{1}_{\left\{\left|D_{n, k}\right|>\epsilon \sigma_{n}\right\}} \mid W_{k-1}\right] \rightarrow^{\mathrm{P}} 0
$$

for every $\epsilon>0$, then

$$
\frac{S_{n}}{\sigma_{n}} \mid W_{0} \stackrel{\mathrm{P}}{\rightarrow} \operatorname{Normal}[0, \lambda] .
$$

Proof. The result follows from the martingale central limit theorem (see, for example, $\left[1\right.$, pp. 475-478]) applied conditionally given $\mathcal{F}_{0}:=\sigma\left(\ldots, W_{-1}, W_{0}\right)$. For $\lambda=1$, the proof is detailed in [13], and the extension to $\lambda \neq 1$ presents no difficulty.

In the next proposition we write $S_{n}=S_{n}(g)$ and $\sigma_{n}=\sigma_{n}(g)$ to emphasize the dependence on $g$. We also use the following result.

Lemma 1. If $Z_{n} \mid W_{0} \stackrel{\mathrm{P}}{\rightarrow} G$ and $Z_{n}^{\prime}-Z_{n} \rightarrow^{\mathrm{P}} 0$, then $Z_{n}^{\prime} \mid W_{0} \stackrel{\mathrm{P}}{\rightarrow} G$.

Proof. The result follows from the unconditional version of Slutzky's theorem, by considering subsequences along which convergence in probability can be replaced by almost-sure convergence.

Proposition 3. If $\ell(n) \rightarrow \infty$ and (10) holds for a given $g$, then, for any $j \geq 1, \sigma_{n}\left(Q^{j} g\right) \sim$ $\sigma_{n}(g)$ and $(10)$ holds with the same $\lambda$ when $g$ is replaced by $Q^{j} g$.

Proof. It suffices to prove the result for $j=1$. In this case, the proof follows from $S_{n}(g)-S_{n}(Q g)=\sum_{k=1}^{n}\left[g\left(W_{k}\right)-Q g\left(W_{k-1}\right)\right]+Q g\left(W_{0}\right)-Q g\left(W_{n}\right)$, which implies that

$$
\begin{aligned}
\left|\sigma_{n}(g)-\sigma_{n}(Q g)\right| & \leq\left\|S_{n}(g)-S_{n}(Q g)\right\| \\
& \leq\left\|g\left(W_{1}\right)-Q g\left(W_{0}\right)\right\| \sqrt{n}+2\left\|Q g\left(W_{0}\right)\right\| \\
& =o\left[\sigma_{n}(g)\right],
\end{aligned}
$$

and Lemma 1.

Remark 1. The proof of Proposition 3 does not use the reversibility and, therefore, is valid for any stationary process.

Remark 2. Proposition 3 illustrates an important difference between the cases $\ell(n) \rightarrow \infty$ and $\ell(n) \rightarrow \kappa$, considered in [7]. For if (2) holds then

$$
\kappa=\kappa(g)=2 \lim _{n \rightarrow \infty} \sum_{k=0}^{n}\left(1-\frac{k}{n}\right)\left\langle g, Q^{k} g\right\rangle-\|g\|^{2} .
$$

It is then not difficult to see that (11) holds when $g$ is replaced by $Q^{j} g$, and that $[\kappa(g)+\cdots+$ $\left.\kappa\left(Q^{n} g\right)\right] / n$ approaches 0 as $n \rightarrow \infty$, by Theorem 2 of [14]. 
Remark 3. Kipnis and Varadhan [7] showed that if (2) holds then $D_{n, k}$ converges in $L^{2}(P)$ for every $k$. Clearly, this is impossible if $\ell(n) \rightarrow \infty$. However, if $D_{n, 1} / \sqrt{\ell(n)}$ converged in $L^{2}(P)$, then (8) and (9) would follow easily with $\lambda=1$, and the conditional distributions of $S_{n} / \sigma_{n}$ would converge to the standard normal distribution, as noted in [13]. This hope cannot be realized either, however, if $\lim _{n \rightarrow \infty} \ell(n)=\infty$. For, $D_{n, 1} / \sqrt{\ell(n)}$ cannot be a Cauchy sequence, in this case. To see this, first observe that

$$
\left\|\frac{D_{n, 1}}{\sqrt{\ell(n)}}-\frac{D_{m, 1}}{\sqrt{\ell(m)}}\right\|^{2}=\frac{1}{\ell(n)}\left\|D_{n, 1}\right\|^{2}+\frac{1}{\ell(m)}\left\|D_{m, 1}\right\|^{2}-\frac{2}{\sqrt{\ell(m) \ell(n)}}\left\langle D_{m, 1}, D_{n, 1}\right\rangle
$$

and

$$
\begin{aligned}
\left\langle D_{m, 1}, D_{n, 1}\right\rangle & =\left\langle\bar{V}_{n} g\left(w_{1}\right)-Q \bar{V}_{n} g\left(w_{0}\right), \bar{V}_{m} g\left(w_{1}\right)-Q \bar{V}_{m} g\left(w_{0}\right)\right\rangle \\
& =\left\langle\bar{V}_{n} g, \bar{V}_{m} g\right\rangle-\left\langle Q \bar{V}_{n} g, Q \bar{V}_{m} g\right\rangle \\
& =\left\langle\bar{V}_{n} g, \bar{V}_{m} g\right\rangle-\left\langle Q^{2} \bar{V}_{n} g, \bar{V}_{m} g\right\rangle \\
& =\left\langle\left(I-Q^{2}\right) \bar{V}_{n} g, \bar{V}_{m} g\right\rangle \\
& =\left\langle\left(V_{2}-\frac{1}{n} Q V_{n} V_{2}\right) g, \bar{V}_{m} g\right\rangle
\end{aligned}
$$

So, for any fixed $m$,

$$
\lim _{n \rightarrow \infty}\left\|\frac{D_{n, 1}}{\sqrt{\ell(n)}}-\frac{D_{m, 1}}{\sqrt{\ell(m)}}\right\|^{2}=1+\frac{1}{\ell(m)}\left\|D_{m, 1}\right\|^{2},
$$

and, therefore,

$$
\lim _{m \rightarrow \infty} \lim _{n \rightarrow \infty}\left\|\frac{D_{n, 1}}{\sqrt{\ell(n)}}-\frac{D_{m, 1}}{\sqrt{\ell(m)}}\right\|^{2}=2 .
$$

\section{Examples}

For a simple reversible chain, let $v$ be a probability measure on the Borel sets of $\mathbb{R}$, let $p: \mathbb{R} \rightarrow(0,1)$ be a measurable function for which

$$
\theta=\int_{\mathbb{R}} \frac{\mathrm{d} v}{1-p}<\infty
$$

and let

$$
Q(w ; B)=p(w) \mathbf{1}_{B}(w)+[1-p(w)] v\{B\}
$$

for Borel sets $B \subseteq \mathbb{R}$ and $w \in \mathbb{R}$. Then $Q$ is a stochastic transition function with stationary distribution

$$
\mathrm{d} \pi=\frac{\mathrm{d} \nu}{\theta(1-p)},
$$

and (1) is satisfied. Thus, there is a reversible Markov chain $\ldots, W_{-1}, W_{0}, W_{1}, \ldots$ with transition function $Q$ and marginal distribution $\pi$. This construction is classical, and is described in [11, pp. 134-135].

Now let $\tau_{0}, \tau_{1}, \tau_{2}, \ldots$ be the times before the process jumps, where

$$
\tau_{0}=\max \left\{n \geq 0: W_{n}=W_{0}\right\} \quad \text { and } \quad \tau_{k}=\max \left\{n>\tau_{k-1}: W_{n}=W_{\tau_{k-1}+1}\right\} .
$$


Then $W_{\tau_{k}}=W_{\tau_{k-1}+1}$, and

$$
S_{\tau_{m}}=\tau_{0} X_{0}+\left(\tau_{1}-\tau_{0}\right) X_{\tau_{1}}+\cdots+\left(\tau_{m}-\tau_{m-1}\right) X_{\tau_{m}} .
$$

By the Markov property, $\left(\tau_{0}, W_{0}\right)$ and $\left[\left(\tau_{j}-\tau_{j-1}\right), W_{\tau_{j}}\right], j \geq 1$, are independent random vectors for which $W_{\tau_{j}} \sim v$ and

$$
\mathrm{P}\left[\tau_{j}-\tau_{j-1} \geq k \mid W_{\tau_{j}}=w\right]=p(w)^{k-1}
$$

for all $w \in \mathcal{W}, k \geq 1$, and $j \geq 1$. It follows that $\mathrm{E}\left[\tau_{j}-\tau_{j-1} \mid W_{\tau_{j}}=w\right]=1 /[1-p(w)]$ and

$$
\mathrm{E}\left[\tau_{j}-\tau_{j-1}\right]=\int_{W} \frac{\mathrm{d} \nu}{1-p}=\theta .
$$

By way of contrast, $W_{\tau_{0}}=W_{0} \sim \pi$, and $\mathrm{E}\left[\tau_{0}\right]=\int p \mathrm{~d} \pi /(1-p)$, possibly infinite. Let $Y_{j}=\left(\tau_{j}-\tau_{j-1}\right) X_{\tau_{j}}$ and $T_{m}=Y_{1}+\cdots+Y_{m}$, so that $S_{\tau_{m}}=\tau_{0} W_{0}+T_{m}$. Then $Y_{1}, Y_{2}, \ldots$ are independent and identically distributed (i.i.d.); moreover, $\mathrm{E}\left[Y_{j}\right]=0$, since

$$
\mathrm{E}\left[\left(\tau_{j}-\tau_{j-1}\right) X_{\tau_{j}}\right]=\mathrm{E}\left[\frac{g\left(W_{\tau_{j}}\right)}{1-p\left(W_{\tau_{j}}\right)}\right]=\int_{W} \frac{g}{1-p} \mathrm{~d} \nu=\theta \int_{W} g \mathrm{~d} \pi,
$$

and $g \in L_{0}^{2}(\pi)$. Let

$$
H(y)=\int_{\left|Y_{j}\right| \leq y} Y_{j}^{2} \mathrm{dP},
$$

and recall the following version of the central limit theorem for i.i.d. variables (with possibly infinite variances); see, for example, [4, pp. 576-578]. If $Y_{1}, Y_{2}, \ldots$ are (any) i.i.d. random variables for which $\mathrm{E}\left[Y_{j}\right]=0$ and $H(y)$ varies slowly at $\infty$, then there are $\gamma_{m}$ for which

$$
\gamma_{m}^{2} \sim m H\left(\gamma_{m}\right) \quad \text { and } \quad \frac{T_{m}}{\gamma_{m}} \stackrel{\mathrm{D}}{\rightarrow} \operatorname{Normal}[0,1] .
$$

The following lemma is intuitive, and the proof is presented after Proposition 4 is established. To state it, define integer-valued random variables $m_{n}$ such that $\tau_{m_{n}} \leq n<\tau_{m_{n}+1}$ for $n=$ $1,2 \ldots$

Lemma 2. As $n \rightarrow \infty, S_{n}-T_{m_{n}}=O_{p}(1)$. If $H$ varies slowly at $\infty$ then $T_{m_{n}}-T_{\lfloor n / \theta\rfloor}=$ $o_{p}\left(\gamma_{n}\right)$.

Proposition 4. If $H(y)$ varies slowly and $\gamma_{m}^{2} \sim m H\left(\gamma_{m}\right)$, then

$$
\frac{S_{n}}{\gamma_{n}} \mid W_{0} \stackrel{\mathrm{P}}{\rightarrow} \text { Normal }\left[0, \frac{1}{\theta}\right] \text {. }
$$

Proof. That $T_{m} / \gamma_{m} \stackrel{\mathrm{D}}{\rightarrow} \operatorname{Normal}[0,1]$ was noted above. So, since $\gamma_{\lfloor n / \theta\rfloor} \sim \gamma_{n} / \sqrt{\theta}, T_{\lfloor n / \theta\rfloor} / \gamma_{n}$ $\stackrel{\mathrm{D}}{\rightarrow} \operatorname{Normal}[0,1 / \theta]$, and since $W_{0}$ and $T_{m}$ are independent for all $m$, the conditional distributions have the same limit. The proposition now follows directly from Lemmas 1 and 2.

Proof of Lemma 2. First observe that $S_{n}-T_{m_{n}}=\tau_{0} W_{0}+\left(n-\tau_{m_{n}}\right) W_{\tau_{m}+1}$. It is clear that $\tau_{0} W_{0}$ is stochastically bounded and that $\left|\left(n-\tau_{m_{n}}\right) W_{\tau_{m_{n}+1}}\right| \leq\left(\tau_{m_{n}+1}-\tau_{m_{n}}\right)\left|W_{\tau_{m_{n}+1}}\right|$. To see that the latter term is stochastically bounded, let $f$ denote the marginal mass function of $\tau_{j}-$ $\tau_{j-1}, j=1,2, \ldots$. Then the asymptotic distribution of $\tau_{m_{n}+1}-\tau_{m_{n}}$ has probability mass 
function $\tilde{f}(k)=k f(k) / \theta$, by the renewal theorem, [4, p. 271], and the conditional distribution of $W_{\tau_{m_{n}+1}}$ given $\tau_{m_{n}+1}-\tau_{m_{n}}$ does not depend on $n$. That $\left(\tau_{m_{n}+1}-\tau_{m_{n}}\right)\left|W_{\tau_{m_{n}+1}}\right|=O_{p}(1)$ follows easily.

The proof of the second assertion uses the following version of Lévy's inequality [9, p. 259]: if $H$ varies slowly at $\infty$ then $K^{-1}:=\inf \left\{\min \left(\mathrm{P}\left[T_{k}<0\right], \mathrm{P}\left[T_{k}>0\right]\right): k \geq 1\right\}>0$, and

$$
\mathrm{P}\left[\max _{k \leq n}\left|T_{k}\right|>t\right] \leq K \mathrm{P}\left[\left|T_{n}\right|>t\right]
$$

for all $t>0$. Observe that

$$
\begin{aligned}
& \mathrm{P}\left[\left|T_{m_{n}}-T_{\lfloor n / \theta\rfloor}\right| \geq \epsilon \gamma_{n}\right] \leq \mathrm{P}\left[\left|m_{n}-\left\lfloor\frac{n}{\theta}\right\rfloor\right| \geq \delta n\right] \\
& +\mathrm{P}\left[\max _{|k \theta-n| \leq \theta \delta n+\theta}\left|T_{k}-T_{\lfloor n / \theta\rfloor}\right| \geq \epsilon \gamma_{n}\right] .
\end{aligned}
$$

The first term on the right-hand side approaches 0 for any $\delta>0$ by the law of large numbers. Letting $N_{n}=\lfloor n \delta / \theta\rfloor+4$ and using (13), the second term is at most $2 K \mathrm{P}\left[\left|T_{N_{n}}\right| \geq \epsilon \gamma_{n}\right]$. So, by the central limit theorem, the limit superior of the right-hand side of (14) is at most $4 K[1-\Phi(\epsilon / \sqrt{\delta})]$, which approaches 0 as $\delta \rightarrow 0$.

For the example below, observe that if $f \in L^{1}(\pi)$ then $Q f(w)=p(w) f(w)+[1-$ $p(w)] \int_{\mathcal{W}} f \mathrm{~d} v$. So, if $\boldsymbol{W}=\mathbb{R}, v$ is a symmetric measure, $p$ is a symmetric function, and $f$ is an odd function, then $Q^{n} f=p^{n} \times f$.

Example 1. Consider (12) with $p(w)=\mathrm{e}^{-1 /|w|}$ and

$$
v\{\mathrm{~d} z\}=\mathrm{e} \frac{[1-p(z)] \mathrm{d} z}{2 z^{2}} \text { for }|z| \geq 1 .
$$

In which case $\theta=\mathrm{e}$ and $\pi\{\mathrm{d} w\}=\mathrm{d} w / 2 w^{2}$ for $|w| \geq 1$. Let $g(w)=\operatorname{sgn}(w)$. Then $g \in L_{0}^{\infty}(\pi), Q^{n} g=p^{n} \times g$, and

$$
\left\langle g, Q^{n} g\right\rangle=\int_{\mathbb{R}} p^{n} \mathrm{~d} \pi=\int_{1}^{\infty} \mathrm{e}^{-n / w} \frac{\mathrm{d} w}{w^{2}}=\frac{1}{n} \int_{0}^{n} \mathrm{e}^{-x} \mathrm{~d} x \sim \frac{1}{n} .
$$

It follows that $\left\langle g, \bar{V}_{n} g\right\rangle \sim\left\langle g, V_{n} g\right\rangle \sim \log (n)$ and $\sigma_{n}^{2}=\left[2\left\langle g, \bar{V}_{n} g\right\rangle-\|g\|^{2}\right] n \sim 2 n \log (n)$. So, (3) is satisfied with $\ell(n) \sim 2 \log (n)$.

Recall the definition of the $\tau_{j}$ and the distribution of $\left[\tau_{j}-\tau_{j-1}, W_{\tau_{j}}\right]$. Then

$$
\mathrm{P}\left[\left|\left(\tau_{j}-\tau_{j-1}\right) X_{\tau_{j}}\right|>k\right]=\mathrm{P}\left[\left(\tau_{j}-\tau_{j-1}\right)>k\right]=\int_{\mathbb{R}} p^{k} \mathrm{~d} \nu=\mathrm{e} \int_{\mathbb{R}}(1-p) p^{k} \mathrm{~d} \pi .
$$

The last integral in the previous display is just

$$
\int_{1}^{\infty}\left(1-\mathrm{e}^{-1 / z}\right) \mathrm{e}^{-k / z} \frac{\mathrm{d} z}{z^{2}}=\frac{1}{k} \int_{0}^{k}\left(1-\mathrm{e}^{-y / k}\right) \mathrm{e}^{-y} \mathrm{~d} y \sim \frac{1}{k^{2}} \int_{0}^{\infty} y \mathrm{e}^{-y} \mathrm{~d} y=\frac{1}{k^{2}}
$$

thus,

$$
\mathrm{P}\left[\left|\left(\tau_{j}-\tau_{j-1}\right) X_{\tau_{j}}\right| \geq k\right] \sim \frac{\mathrm{e}}{k^{2}} .
$$


It follows easily that $H(y) \sim 2 \mathrm{e} \log (y)=\mathrm{e} \ell(y), \gamma_{n}^{2}=2 \mathrm{e} n \log \left(\gamma_{n}\right) \sim \mathrm{e} n \log (n)=\frac{1}{2} \mathrm{e} \sigma_{n}^{2}$, and, therefore,

$$
\frac{S_{n}}{\sigma_{n}} \stackrel{\mathrm{D}}{\rightarrow} \text { Normal }\left[0, \frac{1}{2}\right]
$$

(a nonstandard normal distribution).

Since $\mathrm{E}\left[\sigma_{n}^{-2} S_{n}^{2}\right]$ is bounded, it follows that $\mathrm{E}\left|S_{n}\right| \sim \pi^{-1 / 2} \sigma_{n}$ and, therefore, that $S_{n} / \mathrm{E}\left|S_{n}\right|$ $\stackrel{\mathrm{D}}{\rightarrow}$ Normal $\left[0, \frac{1}{2} \pi\right]$. The latter convergence can also be deduced from Theorem 4 of [10]. To this end, it suffices to verify Equation (3.2) of that paper. Since $|g| \leq 1$, it is not difficult to see that the term whose limit is taken in [10, Equation (3.2)] is at most $\sigma_{n}^{-2} \sum_{k=1}^{n} k \beta_{k}$, where $\beta_{k}$ is the coefficient of absolute regularity. So, it suffices to show that $\beta_{n}$ is of order $1 / n$, and this may be deduced from the equation at the top of page 136 of [11] together with the relation $\mathrm{P}\left[\tau_{0}>n\right]=\int_{\mathbb{R}} p^{n} \mathrm{~d} \pi \sim 1 / n$. (The $\tau$ in [11] is our $\tau_{0}+1$.) Conditional convergence is not asserted in Theorem 4 of [10], but is implicit in the proof; $\mathrm{E}\left|S_{n}\right| \sim \pi^{-1 / 2} \sigma_{n}$ is not deducible from that theorem, however, because $S_{n}$ is not normalized by $\sigma_{n}$ there.

Example 2. A slight modification of Example 1 produces a very simple bounded stationary sequence whose normalized partial sums converge in distribution to a stable distribution. Other examples may be found in [8]. If (15) is changed to

$$
\nu\{\mathrm{d} z\}=\frac{[1-p(z)] \mathrm{d} z}{2 \gamma_{\alpha}|z|^{\alpha}}
$$

for $|z| \geq 1$, where $1<\alpha<2$ and $\gamma_{\alpha}=\int_{0}^{1} y^{\alpha-2}\left(1-\mathrm{e}^{-y}\right) \mathrm{d} y$, then $\pi\{\mathrm{d} z\}=(\alpha-1) /\left(2|z|^{\alpha}\right) \mathrm{d} z$ for $|z| \geq 1$, and

$$
\mathrm{P}[Y>y] \sim \frac{\Gamma(\alpha)}{\gamma_{\alpha} y^{\alpha}}
$$

as $y \rightarrow \infty$. It then follows that $n^{-1 / \alpha} S_{n} \stackrel{\mathrm{D}}{\rightarrow} Z$, where $Z$ has a symmetric stable distribution with characteristic function $\mathrm{e}^{-c_{\alpha}|t|^{\alpha}}$ and $c_{\alpha}=(\alpha-1) \Gamma(\alpha) \int_{0}^{\infty} x^{-\alpha} \sin (x) \mathrm{d} x$.

\section{References}

[1] Billingsley, P. (1995). Probability and Measure, 3rd edn. John Wiley, New York.

[2] Bingham, N. H., Goldie, C. M. and Tuegels, J. L. (1987). Regular Variation. Cambridge University Press.

[3] Cuny, C. And Peligrad, M. (2010). Central limit theorem started at a point for stationary processes and additive functionals of reversible Markov chains. To appear in J. Theoret. Prob. Available at http:// www.springerlink.com/content/f888474451015636/fulltext.pdf.

[4] Feller, W. (1971). An Introduction to Probability Theory and Its Applications, Vol. II, 2nd edn. John Wiley, New York.

[5] Halmos, P. (1957). Introduction to Hilbert Spaces and the Theory of Spectral Multiplicity, 2nd edn. Chelsea, New York.

[6] Kipnis, C. And Landim, C. (1999). Scaling Limits of Interacting Particle Systems. Springer, Berlin.

[7] Kipnis, C. and Varadhan, S. R. S. (1986). Central limit theorem for additive functionals of reversible Markov processes and applications to simple exclusions. Commun. Math. Phys. 104, 1-19.

[8] Koul, H. L. AND Surgailis, D. (2001). Asymptotics of empirical processes of long memory moving averages with infinite variance. Stoch. Process. Appl. 91, 309-336.

[9] Loève, M. (1977). Probability Theory. I, 4th edition, Springer, New York.

[10] Merlevède, F. AND Peligrad, M. (2006). On the weak invariance principle for stationary sequences under projective criteria. J. Theoret. Prob. 19, 647-689.

[11] Rio, E. (2000). Théorie Asymptotique des Processus Aléatoires Faiblement Dépendants (Math. Appl. 31). Springer, Berlin.

[12] Tierney, L. (1994). Markov chains for exploring posterior distribution. Ann. Statist. 22, 1701-1762.

[13] Wu, W. B. AND Woodroofe, M. (2004). Martingale approximations for sums of stationary processes. Ann. Prob. 32, 1674-1690.

[14] Zhao, O. ANd Woodroofe, M. (2008). On martingale approximations. Ann. Appl. Prob. 18, 1831-1847. 